\title{
Die Erfahrungen krebskranker Kinder mit Homöopathie
}

— Pädiater aus Herdecke schickten Fragebögen an alle 1063 Familien mit Kindern, die laut Deutschem Krebsregister im Jahr 2011 an Krebs erkrankt waren. Von dieser Population setzten 367 (35\%) komplementärmedizinische Therapien zusätzlich zur konventionellen Therapie ein. Von dieser Sub-Population verwendeten 45\% homöopathische Mittel.

Der Vergleich der Homöopathienutzer mit denjenigen, die zwar komplementäre Verfahren, aber keine Homöopathie einsetzten zeigte interessante Aspekte: Homöopathienutzer erhofften sich häufiger eine körperliche Stabilisierung oder eine Stärkung des Immunsystems oder eine bessere Toleranz der konventionellen Therapie. Besorgniserregend ist, dass diese Gruppe meist von Heilpraktikern homöopathisch betreut werde, und dass $89 \%$ von ihnen die Homöopathie in ähnlicher Situation erneut einsetzen würden.

Kommentar

Diese Daten implizieren, dass Eltern, die ihre krebskranken Kinder mit Homöopathie behandeln lassen, sich u.a. bessere Heilungs- chancen durch dieses Vorgehen erwarten. Die Evidenz zu diesem Thema zeigt jedoch, dass diese Hoffnung nicht erfüllt werden kann. Die Prinzipien der Homöpathie sind biologisch nicht plausibel und die klinischen Studien belegen die Wirkungslosigkeit der Homöopathie. Falsche Hoffnungen bei Eltern mit krebskranken Kindern zu wecken, ist meines Erachtens nicht nur nicht rational, sondern grausam.

E. ERNST :

\section{- A. Längler et al.}

Use of Homeopathy in Pediatric Oncology in Germany. Evidence-based Complementary and Alternative Medicine. 2011;2011:867151

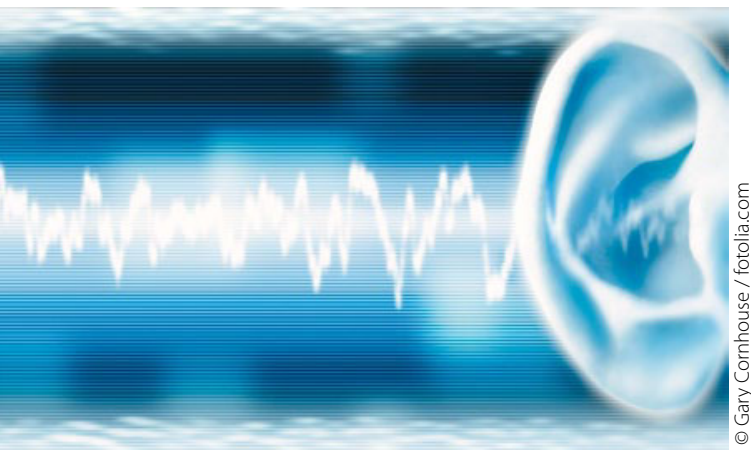

Gibt es Unterschiede in der Prognose, wenn Patienten mit akutem Hörsturz systemisch oder intratympanal mit Kortikosteroiden behandelt werden?

— Der Hörsturz tritt mit einer Inzidenz von 5-20 pro 100000 Personen auf. Am häufigsten kommt dieses Krankheitsbild im Alter zwischen 45 und 55 Jahren vor. Etwa ein Drittel der Patienten weist zusätzlich vestibuläre Symptome auf. Ähnlich wie bei der Neuritis vestibularis wird hier eine Neuritis des achten Hirnnerven vermutet. Deshalb werden Patienten in der Regel mit Kortikosteroiden behandelt. Die hier vorliegende randomisierte Studie verglich die lokale mit der systemischen Steroidtherapie.

\section{Beim akuten Hörsturz Kortikoide einfach oral geben}

Es handelt sich um eine prospektive, randomisierte Nichtunterlegenheitsstudie von 250 Patienten mit einseitigem Hörsturz innerhalb von 14 Tagen nach Beginn der Symptome. Die Studie wurde an 16 Zentren in den USA durchgeführt. Die Patienten wurden über sechs Monate nachbeobachtet. Die Behandlung erfolgte entweder über 14 Tage mit $60 \mathrm{mg}$ oralem Prednison mit langsamem Ausschleichen oder mit vier konsekutiven Injektionen innerhalb von 14 Tagen mit $40 \mathrm{mg}$ Methylprednisolon in das Mittelohr. Der primäre Endpunkt der Studie war die Änderung der Hörfunktion nach zwei Monaten.

121 Patienten wurden systemisch und 129 lokal behandelt. Das mittlere Alter betrug 51 Jahre; Männer waren häufiger betroffen als Frauen. Der Hörverlust war in Dezibel erheblich. In der systemischen Therapiegruppe verbesserte sich das Hören um 30,7 Dezibel verglichen mit 28,7 Dezibel in der Injektionsgruppe. Das Hörvermögen zwei Monate nach der Behandlung betrug 56 versus 57,6 Dezibel. Bei Patienten mit akutem Hörsturz ist demnach eine systemische Kortisonbehandlung genauso wirksam wie die Injektion von Methylprednisolon in das Mittelohr.

$$
\text { Kommentar }
$$

Es ist sehr wertvoll, dass jetzt für entzündliche Erkrankungen einzelner Hirnnerven in zunehmendem Maße prospektive, randomisierte Studien durchgeführt werden. So konnte die Überlegenheit einer Prednisontherapie sowohl für die periphere Facialisparese, die Neuritis vestibularis und den Hörsturz nachgewiesen werden. Die vorliegende Studie zeigt, dass die unkomplizierte orale Therapie mit Prednison, die auch viel preiswerter ist, genauso wirksam ist wie die aufwendige intratympanale Injektion von Methylprednisolon. Dies bedeutet, dass nach Ausschluss anderer Ursachen eines Hörsturzes die Behandlung problemlos im ambulanten Bereich durchgeführt werden kann.

H.-C. DIENER =

- S. D. Rauch et al.

Oral vs intratympanic corticosteroid therapy for idiopathic sudden sensorineural hearing loss: a randomized trial. JAMA 305 (2011) 2071-2079 